Int. J. Curr. Res. Med. Sci. (2017). 3(5): 122-128

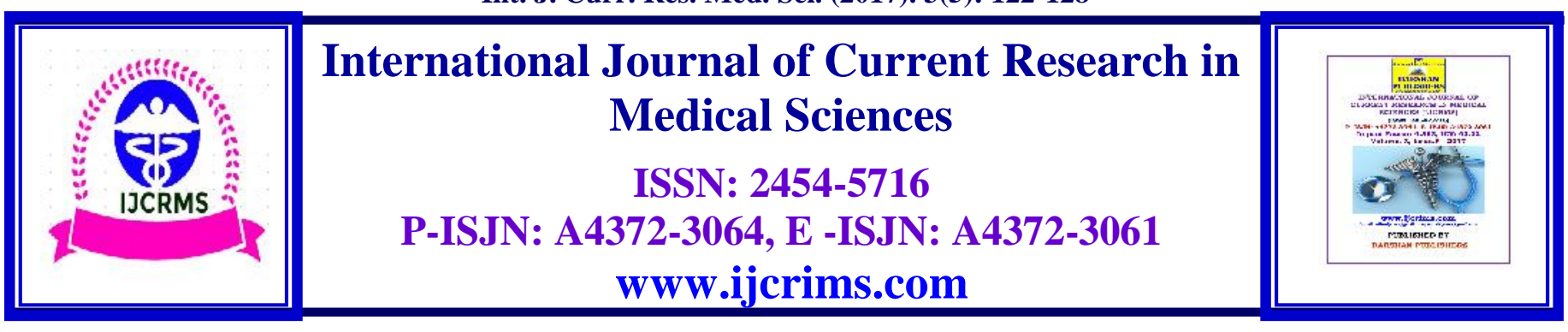

\title{
Prevalence of Acinetobacter infections among Intensive Care Unit's patients in Najran
}

\author{
Dr. Abdullah Aedh \\ Assistant Professor of Internal Medicine at Najran University, College of Medicine and Internal Medicine \\ Consultant at Najran University Hospital and King Khalid Hospital \\ *Corresponding author: nahid1768@ hotmail.com
}

\begin{abstract}
The rising prevalence of Acinetobacter infections in the intensive care units (ICUs) causes a great concern due their extraordinary ability to develop resistance to multiple classes of antimicrobial agents. This is a cross-sectional and facility based study that conducted in King Khalid hospital in Najran, Saudi Arabia. The aim of this study is to estimate the prevalence of Acinetobacter infections in the ICU patients, and to identify antibiotic susceptibility of the Acinetobacter isolates. 180 ICU patients were studied during the period from Aug. 2016 to April 2017. Acinetobacter was isolated in $21(11.7 \%)$ of patients. It was isolated from tracheal secretions in $43 \%$ of the isolates, followed by sputum (23\%), wounds (14\%), Central Venous Line (CVL) (9\%), urine and blood (6\%) for each respectively.

Acinetobacter infection was increased significantly in patients with old ages, patients with chronic diseases and those undergoing invasive procedures $(\mathrm{P}=0.001)$. Acinetobacter sensitivity tests were performed, they showed that the isolates were resistant to many antibiotics in terms of Ampicillin, Cephalothin, Cefuroxime, Gentamycin, Amikacin, Tobramycin, Ofloxacine, Rifampicin and Colistin was respectively $87 \%, 86 \%, 79 \%, 76 \%, 52 \%, 43 \%, 33 \%, 32 \%$ and $8 \%$.

It was concluded that Acinetobacter is an important nosocomial infection pathogen in the ICU patients. Few treatment options are currently available, and for this reason, prevention and infection control measures are essential, including antibiotic control strategies in the ICUs.
\end{abstract}

Keywords: ICU patients, Prevalence, Acinetobacter, antibiotic resistance,

\section{Introduction}

During the last two decades, clinicians in various countries have witnessed a a growing number of critically ill patients who suffer from infections due to microorganisms that belong to the Acinetobacter genus, mainly strains of the species Acinetobacter. ${ }^{1,2}$ Acinetobacter are a group of non-fermentative, Gram-negative cocco-bacillus that has emerged as an important nosocomial pathogen. It is water organism and preferentially colonizes aquatic environments. ${ }^{3}$

When Acinetobacter infections occur, they usually involve organ systems that have a high fluid content (eg, respiratory tract, CSF, 
peritoneal fluid and urinary tract). ${ }^{4}$ They are increasingly implicated as a cause of healthcareassociated infections (HAI), which confer a high risk of morbidity and mortality to patients. ${ }^{5}$

Acinetobacter can cause serious and sometimes life-threatening infections. It is an opportunistic pathogen known for its intrinsic resistance to antibiotics and greater ability to rapidly acquire resistance genes as mobile genetic elements (plasmids, transposons, integrons cassettes and insertion sequences). ${ }^{6,1}$ In addition, their ubiquitous nature in the ICU environment coupled with inadequate infection-control practices have continuously raised the incidence of Acinetobacter infections over the past two decades. ${ }^{7}$

The precise mechanisms that explain how multiple-drug resistance occurs are not fully known. However, recent studies have shown that Acinetobacter can produce great diversity of chromosomal and plasmid-mediated enzymes. ${ }^{8}$ They can produce aminoglycoside-modifying enzymes to neutralize aminoglycosides and thus become resistant to this class of antimicrobial agents. B-lactamases are another type of modifying enzymes that give them potential to become resistant to penicillins, cephalosporins, and carbapenems. ${ }^{9}$ More interestingly, they can also diminish uptake of antibiotics into their cells by either changes in the outer membrane porins to decrease permeability to the agents or by creating active antimicrobial efflux systems. ${ }^{10}$

Risk factors for multi-drug resistant Acinetobacter colonization and infection include prolonged length of hospital stay, exposure to central venous catheterization, urinary catheterization, prior exposure to strong antimicrobials, greater severity of illness, surgery and receipt of invasive procedures. ${ }^{3,8,11}$ Although, risk factors for antibiotic-resistant Acinetobacter infection have been explored in many patient populations, fewer studies have assessed potential differences in risk factors for those infected with antimicrobialresistant versus susceptible strains. ${ }^{4,12}$

\section{Material and Methodology}

This study was performed at King Khalid hospital in Najran city-Saudi Arabia, a tertiary teaching hospital. The study population consists of 180 patients who were admitted in the intensive care unit. This population of patients comprises of patients who have had surgery, on ventilators or intubation, beside prior history of antibiotic usage.

\subsection{Methods of data collection}

Verbal informed consent was obtained from spouse, parents or caregiver of each subject. Therefore, relevant medical history, sociodemographic data and other information obtained from the care giver and case files were entered into a semi-structured close-ended questionnaire.

\subsection{Specimen collection and analyses}

Tracheal aspirate, sputum, blood and urine specimens were collected from all recruited subjects who spent at least 72 hours in the ICU for microscopy, culture and sensitivity tests. Specimens were collected using aseptic technique to prevent contamination. For optimal results, specimens were collected in clean sterile, wide bore containers.

Isolation of Acinetobacter was performed on blood agar and Herellea agar. Identification of clinical isolates were performed by classical bacteriological techniques (direct examination, biological test of orientation). Cultural characteristics observed after an incubation at $36^{\circ} \mathrm{C}$ for 24 hours.

The study of antibiotic susceptibility was performed by the disc diffusion method on Mueller -Hinton agar plates that contained Ampicillin $(10 \mu \mathrm{g})$, Cephalothin (30 $\mu \mathrm{g})$, Cefuroxime $(30 \mu \mathrm{g})$, gentamycin $(10 \mu \mathrm{g})$, Amikacin $(30 \mu \mathrm{g})$, Tobramycin $(10 \mu \mathrm{g})$, Ofloxacin $(5 \mu \mathrm{g})$, Rifampicin $(10 \mu \mathrm{g})$ and Colistin $(5 \mu \mathrm{g})$. 


\subsection{Data analysis}

All data were analyzed using Statistical Package for Social Science (SPSS) version 18.0. Data were presented using frequency tables, charts, as appropriate and cross tabulation to study relationships and association between variables. Statistical significance was set at 5\%.

\section{Results}

A total of 180 patients were recruited to participate in the current study over a period of 9 months, from Aug. 2016 to April 2017. The age of the participants ranged from 11 to 82 years old. The mean patients' age was $46.6 \pm 15.3$ years. The median duration of ICU stay was 5 days (range, 1-170 days).

Majority of the patients (31.67\%) were falling in 4155 years age group, while the $10-25$ years age group constituted the least age group (10.56\%). There were 98 male $(54.4 \%)$, and $82(45.6 \%)$ females, giving a male to female ratio of 1.2:1.

One hundred twenty four $(68.89 \%)$ of the patients were admitted from the emergency department (ER). While the rest were from other wards in the hospitals. More than $43 \%$ of the admitted cases are having chronic illnesses and almost 57\% of them had past history of hospitalization (table 1).

Table (1): Represents socio-demographic characteristics of the selected sample ( $\mathrm{n}=180)$

\begin{tabular}{cccc}
\hline Variable & Category & Frequency & \% \\
\hline Sex & Male & 98 & $54.4 \%$ \\
& Female & 82 & $45.6 \%$ \\
\hline \multirow{4}{*}{ Age in years } & $10-25$ & 19 & $10.56 \%$ \\
& $26-40$ & 32 & $17.78 \%$ \\
& $41-55$ & 57 & $31.67 \%$ \\
Source of admission & $56-70$ & 42 & $23.33 \%$ \\
& $71-85$ & 30 & $16.67 \%$ \\
\hline \multirow{3}{*}{ Causes of admission } & Emergency department (ER) & 124 & $68.89 \%$ \\
& Other departments in the & 56 & $31.11 \%$ \\
& hospital & & \\
\hline Previous & Chronic illnesses & 78 & $43.33 \%$ \\
hospitalization & Surgical operations & 40 & $22.22 \%$ \\
& Accidents & 62 & $34.44 \%$ \\
\hline
\end{tabular}

The result of the laboratory studies shows that 55 cases $(30.6 \%)$ were detected positive for various microbial agents in terms of Acinetobacter, Pseudomonas, E-coli, Staphylococci, Klebsiella pneumonia, Fungus and others. Within the 55 positive patients $44(80 \%)$ had underlying chronic diseases.
Out of the 180 patients studied, Acinetobacter spp was isolated from 21(11.7\%) patients giving a prevalence rate of $11.7 \%$. It was isolated from tracheal secretions in $(42,86 \%)$, followed by sputum (22.86\%), wounds (14.29\%), central venous line $(\mathrm{CVL})$ in $(8.57 \%)$, urine $(5.71 \%)$ and blood $(5.71 \%)$ as displayed in figure (1). 
Figure (1): displayed the sites from where specimens were extracted among positive cases $(n=21)$

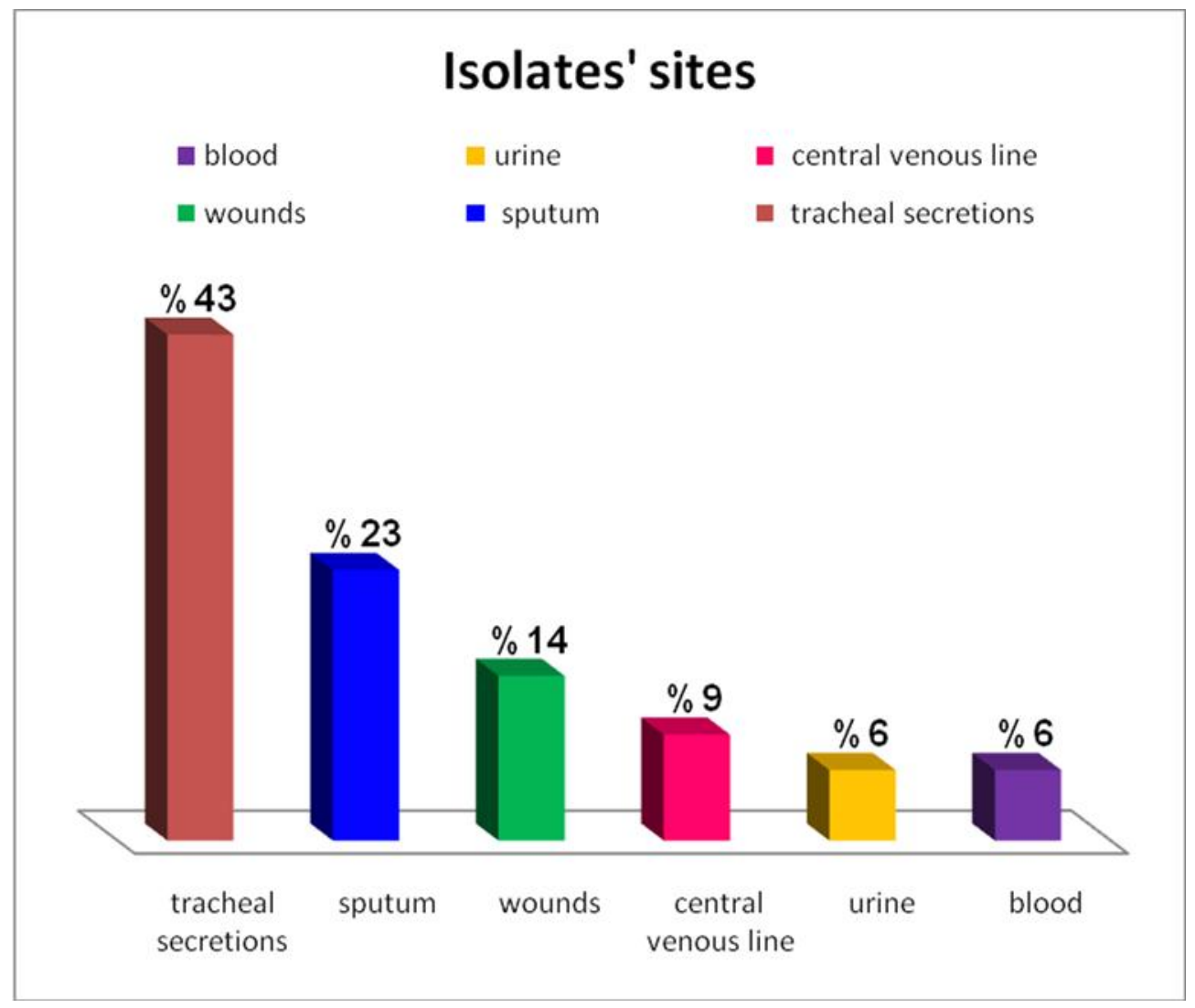

Table 2 shows risk factors related to prior antimicrobial therapy. The average number of prior antimicrobials used was 3.8 in bacteremic patients and 2.7 in non-bacteremic patients $(P<0.001)$.

Table (2): Shows risk factors related to prior antimicrobial therapy

\begin{tabular}{cccccc}
\hline \begin{tabular}{c} 
Mean standard deviation $\begin{array}{c}\text { Non- } \\
\text { of prior exposure to } \\
\text { antibiotics }\end{array}$ \\
\cline { 2 - 6 }
\end{tabular} & $\begin{array}{c}\text { Bacteremic } \\
\text { bacteremic } \\
(\mathbf{n = 1 2 5})\end{array}$ & $\begin{array}{c}\text { Odds } \\
\text { Ratio }\end{array}$ & 95\% CI & $\begin{array}{c}\boldsymbol{P} \text { - } \\
\text { value }\end{array}$ \\
\hline $2.7 \pm 1.1$ & $3.8 \pm 1.4$ & 2.09 & $1.6-$ & $<0.001$ \\
\hline
\end{tabular}

Acinetobacter sensitivity tests were performed, in which the isolates were resistant to many antimicrobial. They showed high resistance to Ampicillin (87.36\%), followed by Cephalothin
(86.64\%), Cefuroxime (79\%), Gentamycin (75.6\%), Amikacin (51.7\%), Tobramycin (42\%), Ofloxacin (32.7\%), Rifamicin (31.1\%) and Colistin (8\%) respectively. (table3). 
Int. J. Curr. Res. Med. Sci. (2017). 3(5): 122-128

Table (3): shows Acietobacter sensitivity tests $\quad(n=21)$

\begin{tabular}{cc}
\hline Antibiotic agent & Resistance (\%) \\
\hline Ampicillin & $87.36 \%$ \\
Cephalothin & $86.64 \%$ \\
Cefuroxime & $79 \%$ \\
Gentamycin & $75.6 \%$ \\
Amikacin & $51.7 \%$ \\
Tobramycin & $42 \%$ \\
Ofloxacin & $32.7 \%$ \\
Rifamicin & $31.1 \%$ \\
Colistin & $8 \%$ \\
\hline
\end{tabular}

\section{Discussion}

In recent years, Acinetobacter has become an important pathogen especially in the ICUs. Patients admitted to ICUs have been shown to be at particular risk of acquiring nosocomial infection with a prevalence rate as high as $30 \% .^{13}$ Several studies on the epidemiological and clinical features of Acinetobacter infections have been published. ${ }^{5,11,12}$

In the current study, Acinetobacter Spp prevalence rate was $11.7 \%$ in the ICU. This prevalence is near to similar studies carried out by Jang et al who reported $12 \%$, Joly who reported $9 \%$, and Ugochukwu who reported $14 \%$ respectively. $6,13,14$ This result in our study is higher than what concluded by Iregbu et al who reported a prevalence of $4.6 \%$ in Lagos. ${ }^{15}$ The majority of positive samples in this study were isolated from the respiratory tract, this finding was in consistent with studies carried out by Garnacho et al. ${ }^{16}$ and Ugochukwu et al. ${ }^{14}$ This high rate of recovery from the respiratory tract may be due to the invasive procedures that are carried out during the process of maintaining airways.

Moreover, the recovery rate of Acinetobacter Spp from urinary tract was almost $6 \%$, this rate is slightly higher than what was concluded by Playford et al who reported $3.2 \% .^{17}$ and $1 \%$ as reported by Ugochukwu et al. ${ }^{14}$ who suggested that this finding confirms the popular report that Acinetobacter is no longer a common uropathogen.
The incidence of Acinetobacter blood stream infection in this study almost $6 \%$ which is higher than what reported by Falagas et al. ${ }^{18}$ who reported $2 \%$ and Ugochukwu et al. ${ }^{14}$ who reported $1.3 \%$, while it was slightly lower than Maragakis and Perl. ${ }^{19}$ who reported $8.8 \%$ incidence in blood.

In the present study, Acinetobacter infection were higher in the patients above 50 years old, patients undergoing chronic diseases and patients that undergoing invasive procedures. This fact in agree with finding of Amini et al. ${ }^{20}$ In contrast, in the current study it was concluded that there was no differences in relation to surgical operations and current antibiotic therapy.

The recent emerging of drug resistant Acinetobacter has caused a great concern worldwide. In the present study, most of Acinetobacter isolates were highly resistant to Ampicillin (87\%), Cephalothin (86.64\%), Cefuroxime (79\%), Gentamycin (76\%), Amikacin (51.7\%), Tobramycin (42\%), Ofloxacin (32.7\%), Rifampicin (31.1\%) and Colistin (8.21\%).

Navon-Venezia et al showed that most of Acinetobacter isolates were resistant to all standard antibiotics. ${ }^{21}$ Other studies concluded that up to $80 \%$ of Acinetobacter isolates were resistant to all aminoglycosides. ${ }^{14,15}$ Moreover, other studies reported Imipenem sensitivity in $89 \%$ and up to $70 \%$ were sensitive to $\beta$-lactam and $\beta$-lactamas inhibitor combination. ${ }^{21,22}$ 
Int. J. Curr. Res. Med. Sci. (2017). 3(5): 122-128

\section{Conclusion and Recommendation}

There has been increasing concern regarding the rise Acinetobacter infections among critically ill patients. They most frequently involve the respiratory tract of intubated patients.

Acinetobacter infection was increased significantly with old ages, patients with chronic underlying diseases, and among patients who undergoing invasive procedures.

As far as resistance to Colistin was estimated in this study to $8 \%$, therefore, Colistin can be considered one of the most effective treatment option for Acinetobacter infection.

The emergence resistant strains of Acinetobacter infections has led to fewer treatment options. Due to these limited therapeutic options, prevention and infection control measures are essential, including not only traditional measures but also antibiotic control strategies in the ICUs. Moreover, to prevent the occurrence and transmission of this organism in the ICUs, infection control measures crucial. Measures described in the clinical trials include hand disinfection by using either alcohol hand-gel or Chlorhexidine. ${ }^{23}$ Barrier and contact precautions such as wearing gloves, gowns, and masks are so essential. Additionally, restricted use of third generation cephalosporins along with other antibiotics control strategy could be useful.

\section{Acknowledgements}

I am indebted to numerous colleagues especially professor Mohamed Ali and dr. Hussain for their valuable contributions in analyzing specimens as well as reviewing the entire work. Thanks are also due to ICU staff for their endless efforts.

\section{References}

1. Horan TC, Andrus M, Dudeck MA: CDC/NHSN surveillance definition of health care-associated infection and criteria for specific types of infections in the acute care setting. American
Journal of Infection Control . 2008; 36(5):309 332.

2. Hartzell JD, Kim AS, Kortepeter MG, Moran KA: Acinetobacter pneumonia; a review. Med Gen Med . 2007; 9(3): 4-4.

3. Cetin ES, Durmaz R, Tetik T, Otlu B, Kaya S, Caliskan A: Epidemiologic characterization of nosocomial Acinetobacter baumannii infections in a Turkish university hospital by pulsed-field gel electrophoresis. Am J. Infect Control. 2009; 37(1): $56-64$.

4. Turton JF, Woodford N, Glover J, Yarde S, Kaufmann ME, Pitt TL: Identification of Acinetobacter baumannii by detection of the blaOXA-51-like carbapenemase gene intrinsic to this species. J Clin Microbiol. 2006; 44(8): 2974 -6 .

5. Chang H, Tang C, Hsu Y, Wan L, Chang Y, Lin C, Tseng Y, Lin Y, Sheu J, Chang Y, Ho M, Lin C. Ho C, Lai C: Nosocomial outbreak of infection with multidrug-resistant Acinetobacter baumannii in a medical center in Taiwan. Infection control and hospital epidemiology. 2009; 30(1): $34-38$.

6. Jang TN, Lee SH, Huang CH, Lee CL, Chen WY: Riak factors and impact of nosocomial Acinetobacter baumannii bloodstream infections in the adult intensive care unit: a case-control study. The journal of hospital infection. 2009; 73(2): $143-150$.

7. Livermore DM, Hill RL, Thomson H, Charlett A, Turton JF, Pike R et al. Antimicrobial treatment and clinical outcome for infections with carbapenem and multiply-resistant Acinetobacter baumannii around London. Int J Antimicrob agents. 2010; 35(1): 19 -24.

8. Wareham DW, Bean DC, Khanna P, Hennessy EM, Krahe D, Ely A, Millar M: Bloodstream infection due to Acinetobacter spp: epidemiology, risk factors and impact of multidrug resistance. European journal of clinical microbiology and infectious diseases. 2008; 27(7): $607-612$.

9. Erbay A, Idil A, Gzel NG, Mumcuoglu I, Balaban $\mathrm{N}$ : Impact of early appropriate antimicrobial therapy on survival in Acinetobacter baumannii bloodstream infections. International journal of antimicrobial agents. 2009; 34(6): 575 - 579. 
10. Zarilli $\mathrm{R}$, Crispino $\mathrm{M}$ and Bagattini $\mathrm{M}$ : Molecular epidemiology of sequential outbreaks of Acinetobacter baumannii in ICU shows the emergence of Carbapenem resistance. J Clin Microbiol. 2004; 42(1): 946 - 53.

11. Young LS, Sabel AL, Price CS: Epidemiologic, clinical, and economic evaluation of an outbreak of clonal multidrug-resistant Acinetobacter baumannii infection in a surgical intensive care unit. Infection control and hospital epidemiology. 2007; 28(11): 1247 - 1254.

12. Mussi M, Gaddy J, Cabruja M, Arivett B et al: The Opportunistic Human Pathogen Acinetobacter baumannii Senses and Responds to light Journal of Bacteriology. 2010; 192(24): $6336-6345$.

13. Joly - Guillou ML: Clinical impact and pathogenecity of Acinetobacter Clin. Microb. Infect. 2005; 11(1): 86873.

14. Ugochukwu NV, Adetona FS, Adeala F, Ajani BR and Olusanya O: Nosocomial Acinetobacter infections in Intensive Care Unit. Am. J. Infect Dis. 2013 ; 9(2): 40 - 45.

15. Iregbu K, Ogunsola $F$, Odugbemi T. Infections caused by Acinetobacter spp. and their susceptibility to 14 antibiotics in Lagos university teaching hospital. Lagos. West Af. J Med. 2002; 21(3): $226-229$.

16. Garnacho-Montero J, Ortiz-Leyba C and Fernadez-Hinojosa et al. Acinetobacter baumannii ventilator associated pneumonia: epidemiological and clinical findings, Intensive Care Med. 2005; 31(1): 649 - 55.
17. Playford EG, Craig JC, Iredell JR: Carbapenemresistant Acinetobacter baumannii in intensive care unit patients: Risk factors for acquisition, infection and their consequences. The Journal of hospital infection. 2007; 65(3): $204-211$.

18. Falagas ME, Bliziotis IA, Siempos I. Attributable mortality of Acinetobacter baumannii infections in critically ill patients: a systematic review of matched cohort and casecontrol studies. Critical care. 2006; 10(2): 48 48.

19. Maragakis L, and Perl TM. Acinetobacter baumannii: epidemiology, antimicrobial resistance, and treatment options. Clinical infectious diseases. 2008; 46(8): 1254- 1263.

20. Amini M, Davati A and Golestanifard M: Frequency of nosocomial infections with antibiotic resistant strains of Acinetobacter spp. in ICU. Iran J Pathol. 2010; 7(4): 241 - 45.

21. Navon-Venezia S, Leavitt A and Carmeli Y. High tigecycline resistance in multidrug-resistant Acinetobacter baumannii. J Antimicrob Chemother. 2007; 59(4): $772-2$.

22. Kwon KT, Oh WS, Song J, Chang H, Jung S, Kim S, Ryu SY, Heo ST, Jung DS, Rhee JY et al: Impact of imipenem resistance on mortality in patients with Acinetobacter bacteraemia. The Journal of Antimicrobial Chemotherapy. 2007; 59(3): $525-530$.

23. Garcia-Garmendia JL et al. Risk factors for Acinetobacter baumannii nosocomial bacteremia in critically ill patients: a cohort study. Clinical Infectious Diseases. 2001;33:939-946.

\begin{tabular}{|c|l|}
\hline \multicolumn{2}{|c|}{ Access this Article in Online } \\
\hline & \\
\hline & Website: \\
\hline Quick Response Code & Www.ijcrims.com \\
\hline
\end{tabular}

How to cite this article:

Abdullah Aedh. (2017). Prevalence of Acinetobacter infections among Intensive Care Unit's patients in Najran. Int. J. Curr. Res. Med. Sci. 3(5): 122-128.

DOI: http://dx.doi.org/10.22192/ijcrms.2017.03.05.017 